\title{
Commentary
}

Graham R.D. Jones* and Michael Legg

\section{Report formatting in laboratory medicine - a call for harmony}

https://doi.org/10.1515/cclm-2017-1165

Received December 13, 2017; accepted March 12, 2018; previously published online April 19, 2018

Abstract: The results of medical laboratory testing are only useful if they lead to appropriate actions by medical practitioners and/or patients. An underappreciated component of the medical testing process is the transfer of the information from the laboratory report into the reader's brain. The format of laboratory reports can be determined by the testing laboratory, which may issue a formatted report, or by electronic systems receiving information from laboratories and controlling the report format. As doctors can receive information from many laboratories, interpreting information from reports in a safe and rapid manner is facilitated by having similar report layouts and formats. Using Australia as an example, there is a wide variation in report formats in spite of a body of work to define standards for reporting. In addition to standardising of report formats, consideration needs to be given to optimisation of report formatting to facilitate rapid and unambiguous reading of the report and also interpretation of the data. Innovative report formats have been developed by some laboratories; however, wide adoption has not followed. The need to balance uniformity of reporting with appropriate innovation is a challenge for safe reporting of laboratory results. This paper discusses the current status and opportunity for improvement in safety and efficiency of the reading of laboratory reports, using current practise and developments in Australia as examples.

Keywords: pathology reporting; patient safety; standardisation.

\footnotetext{
*Corresponding author: Graham R.D. Jones, Department of Chemical Pathology, SydPath, St Vincent's Hospital, Sydney, NSW, Australia; and University of NSW, Sydney, NSW, Australia, E-mail: Graham.jones@svha.org.au Michael Legg: Michael Legg and Associates, University of Wollongong, Bulli, New South Wales, Australia; and Faculty of Engineering and Information Science, University of Wollongong, Wollongong, New South Wales, Australia
}

\section{Introduction}

Significant harm is being done and potential wellness lost because of diagnostic error, but it is underappreciated. In their major review, improving diagnosis in health care, the institute of medicine concluded "that all of us will likely experience a meaningful diagnostic error in our lifetime" [1].

Although data are sparse, radiology and laboratory medicine contribute to diagnostic error, and the evidence suggests that most of this error occurs at the prelaboratory and postlaboratory interfaces [2]. Diagnostic error can be classified into no-fault errors, system-related errors and cognitive errors. In root cause analysis of one series of cases with significant diagnostic error, 5.9 errors per case were found, $65 \%$ of these were system-related errors (most being organisational flaws in policy and procedures and communication) and $74 \%$ were cognitive errors [3].

The transfer of medical information is a point of risk in patient management. If a piece of information is not received as intended by the sender, medical mistakes can occur. This may be especially common in oral communication, or with handwritten notes, and may occur in any medical setting. Examples include handover between medical shifts, prescribing medication and communication of laboratory results. The transfer of information from a laboratory report into a doctor's brain should be a straightforward process; however, even this can be a source of miscommunication. If the design of the reports contributes to an error in information transfer, this can be seen as a "system-related" error in the models of Graber et al. [3]. In general, there seem to be two approaches to improving information communication in this setting. The first is to standardise the reporting format so that a doctor is familiar with the formatting, irrespective of the source. The second is to redesign the reports to optimise transmission of the key facts, possibly using graphical or other techniques. These approaches should not be seen as mutually exclusive; however, standardisation tends to embed current practise and novel report displays tend to be local developments. This paper addresses the risk of miscommunication of routine clinical laboratory results 
in a paper or electronic format. Although the concepts are likely to be relevant to medical settings elsewhere, the paper will focus on the Australian medical environment with specific attention to numerical pathology results.

\section{The situation}

Clinical laboratory results are generally reported from laboratory information systems (LIS) in one of two ways. These are a rendered format, which may be delivered and displayed in either a paper or electronic environment, or an "atomic" format, where the results are transmitted in a structured electronic message with display determined by the receiving system. In the former, the message sender (pathology laboratory) is responsible for the formatting, and in the latter it is the system receiving the message that determines the formatting for the display of the information. A paper report or electronic display of a pdf report would be examples of reports formatted by the testing laboratory.

Pathology reports may consist of results that are largely textual, e.g. histopathology, or largely numerical, e.g. clinical chemistry. The formatting is based on providing the data in an ordered manner, often in a way that would still be possible to display with old technology such as paper and a dot matrix printer, with less focus on using the formatting to transfer the meaning of the data. The use of graphs, colours or other visual signals to facilitate communication of the meaning of the data is generally quite limited.

There are a number of key factors that must be included in a laboratory report of any sort to ensure the linking of the report to the patient and communicate details of the results [4]. These include the patient identifiers, such as patient name, date of birth, sex, address and/ or a medical record number; the date and time of sample collection; the test name(s), the result(s) and associated measurement units; and a reference interval or clinical decision point if relevant. Additionally, a report may contain interpretive data and previous results for the same tests to facilitate interpretation.

\section{Current reports - the risks}

Medical practitioners are often under time pressure and need to assess a wide amount of information to make appropriate medical decisions. This may be reviewing laboratory results in a time critical setting such as the emergency department or operating theatre, or when reviewing results from multiple patients in a single sitting. In either of these settings, the formatting of the report, whether paper or electronic, is important to facilitate the process. Errors may include reviewing the wrong report due to variation in the formatting of the patient identifiers (e.g. James, David; David James; James David), misreading the results as the sequence of results or sample collection dates/times down or across the page is unexpected, the use of different units of measurement, not seeing an attached comment (e.g. noting a change in a previously issued result or issuing an interpretive comment) or failure to recognise a flag indicating an out-of-range result. A study from 2016 has shown that there is wide variation in all these factors in reports from Australian laboratories [5]. In this study, there was variation between laboratories, as well as from a recently published guideline on result reporting [6].

The chances of misreading a report are increased when reports with different formatting are viewed by the same doctor. This may be considered a particular risk in Australia as patients outside hospitals are free to have pathology samples collected and analysed at the laboratory of their choice. This has the effect that a doctor may have reports from different pathology providers, including from public or private hospitals following an admission, with the associated variation in report formatting. This risk may be considered to be increasing significantly with the introduction of laboratory reports into a national e-health record [7], where reports, in pdf formats, will be uploaded from all locations, including hospital inpatient and outpatient, general practise and specialist doctors. In addition, these reports from different pathology providers will be viewed by patients, ancillary clinical services such a dieticians and physiotherapists as well as doctors. In this environment, the risk of misreading data from different reports is likely to be increased.

\section{Possible solutions}

A key solution would seem to be standardisation of the report format [8]. As stated above, there are now Australian guidelines for these factors [6] but with work still to be done to achieve a standardised report [5]. The Australian standard for electronic pathology messaging has required a rendered version of the laboratory report as part of the message since 2012 [9]. This can be in XHTML, RTF, HL7 text, PDF or in a homegrown format called PIT. Despite having been deprecated for some years, PIT is still in 
wide use in Australia, and this is a lesson for standardisation. PIT was first developed in the 1990s before HL7 was adopted in Australia. Since 1998, it has been indicated that it should not be used; however, it takes a very long time to change a "standard" once it is in wide use, with it remaining in common use today. HL7 Australia has recently published a revised messaging standard that draws on the RCPA work and provides, for the first time, everything needed to programme a conformant message capable of semantic interoperability. It is expected that in due course, this will be taken up as a requirement for Australian laboratory accreditation.

There has been an agreement to use pdf formatting for clinical laboratory reporting into the Australian My Health Record [10], which leaves control of formatting in the hands of the testing laboratories and is consistent with the requirement for rendered reports to be sent to general practitioners' desktop software in addition to any supplied atomic data. By contrast, within hospitals, it is more common to send numeric results as discrete pieces of information (atomised), which are rendered into a formatted report by the receiving software, although rendered reports from these locations will be uploaded to the national record. The key driver for the rendered report is the requirement for a fully controlled report format as there is not yet an agreed process for displaying atomised results in receiving systems nor is there uptake of the standards that would allow for safe comparison of results.

In contrast to the within-hospital environment, where the same organisation often controls both the message sending and the message receiving software, allowing testing and communication about requirements and updates, sending messages to external systems creates additional challenges. These have been recognised in an important document from the Office of the National Coordinator for Health Information Technology in the USA [11]. This document provides a checklist for 23 items related to safe transfer of laboratory results in the area of health IT, covering implementation, use and monitoring of IT systems used for test result delivery. A working group as part of the RCPA in Australia has reviewed this document, and its recommendations are included in the RCPA Standards for Pathology Informatics in Australia [6]. This document covers all aspects of formatting and electronic transfer of laboratory results (section 10 of document) covering formatting, communication, testing, system failures, report updates, transfer to third parties and report security. The development of standards is a prerequisite component of standardisation; however, implementation and confirmation requires promotion or regulation. There is also a need for a testing system to identify variation between reports from different laboratories, an "External Quality Assurance" for report formatting, such as foreshadowed in a manual approach by the RCPAQAP [5].

\section{Alternative data presentations}

It has been recognised that the traditional format of laboratory reports is data rich but may be difficult to understand without a strong medical knowledge and a careful reading of the report details. As stated above, there have been a number of proponents of more graphical formats, both in the medical literature $[12,13]$ and in the wider public arena [14]. Proposed tools include the use of graphical outputs with colour shaded areas to indicate risk [12, 13], miniature graphical tools (sparklines) [12] and tools to highlight changes within an individual in addition to as well as absolute values of results [15]. There is no doubt that the "dry" data of a standard numerical report are focussed on data transfer rather than information transfer. There do however seem to be some limitations to the widespread adoption of more graphical formats.

\section{Limitations to alternative formats}

Numerical laboratory reports are often information dense, containing results for up to 30 or more tests, with up to three or more previous results for each of them. Additionally, it is often unclear at the time of reporting results which will be the most important clinically. Indeed a negative result may the key factor a doctor is seeking to make the next medical decision. Because of this, the information needs to be on as few pages (or screens) as possible to minimise page turning (or scrolling) for information. This also creates a difficulty for laboratories issuing reports aimed at highlighting the most important results (or at least allocating extra space on the report to this task), as the importance may not be known.

Although the use of colour has been suggested to improve readability, it is also a potential problem as reports may be reprinted in settings where a colour printer is not available, and colour is also lost if a report is transmitted by fax.

An additional problem is that test results can be put to multiple uses. As a basic example, prostate-specific antigen (PSA) is used for both for case finding and monitoring of prostate cancer. A "low normal" result (within the lower half of the reference interval) in a patient with a previous prostatectomy will indicate likely cancer recurrence 
and be a poor prognostic sign. The same result in a man with a normal prostate may indicate a low risk of prostate cancer. The colour chart in O'Connor [12] addresses this with the heading for the graph "Diabetes test: how is my diabetes?", but information on the reason for the test is required. Although this is not inherently different from any interpretive comment, a text-based comment can explain differences, whereas different graphics that look similar but carry different information may be confusing.

A graphic, e.g. sparklines, or specific flag for a clinically relevant change may be particularly valuable to present information on result changes over time to a clinician used to the system. However, for someone not familiar, there may be increased risk of confusion or misinterpretation.

\section{Combining the issues}

Standardisation of reporting formats, especially at a national level, requires processes of decision making, informing and monitoring. The development of local innovative ways of improving laboratory reporting runs counter to a standardised process, which is likely to be slow moving and aimed somewhat at the lowest common denominator (i.e. what is achievable by all or most computer systems). The challenge is to encourage improvements, subject them to evaluation, and then to adopt them more widely if possible. This is specifically mentioned in the Standards for Pathology Informatics in Australia [6]: "The intention, however, is not to stifle innovation in presentation and so only those aspects of rendering should be considered for adoption where there is a concern around safety and broad support for standardisation".

\section{The elephant in the room}

There will be many readers who, we are sure, will scoff at the discussions above about pdf and other static rendered formats, and point out the obvious advantages of electronic formats for displaying results. In many ways, however, the issues are the same, i.e. that we need agreed formats for result display if doctors and patients move between different pathology providers. This applies to additional supporting features such as real-time graphical analysis, "hover buttons" for further test information or accessing data from expert systems. In the electronic resulting scenario, the need is for the manufacturers of data-receiving software to work together to provide a consistent and safe format.

\section{Conclusions}

Many millions of laboratory reports are produced every year which are, and will be, viewed by many people with different medical literacy. The challenge is to report these results in ways that minimise the risk of miscommunication and misinterpretation, in whatever format is used for the process. Progress in this work will involve laboratory scientists and pathologists, IT and communication specialists, practising doctors and patient representatives, as well as a structure to continue the development and implementation of safe reporting.

Kahneman and Tversky [16] have shown that we respond to how something is put not what is put. This is both a safety issue and an opportunity. Much more could be done by using the communication of the results of laboratory testing to make the job of the recipient easier and more likely to improve health outcomes.

Author contributions: All the authors have accepted responsibility for the entire content of this submitted manuscript and approved submission.

Research funding: None declared.

Employment or leadership: None declared.

Honorarium: None declared.

Competing interests: The funding organization(s) played no role in the study design; in the collection, analysis, and interpretation of data; in the writing of the report; or in the decision to submit the report for publication.

\section{References}

1. Balogh EP, Miller BT, Ball JR, editors. National Academies of Sciences, Engineering, and Medicine: Improving diagnosis in health care. Washington, DC: The National Academies Press, 2015.

2. Khoury M, Burnett, Mackay MA. Error rates in Australian chemical pathology laboratories. Med J Aust 1996;165:128-30.

3. Graber ML, Franklin N, Gordon R. Diagnostic error in internal medicine. Arch Intern Med 2005;165:1493-9.

4. National Pathology Accreditation Advisory Council (NPAAC) 2013 Requirements For Medical Pathology Services (First Edition 2013).

5. Koetsier S, Jones GR, Badrick T. Safe reading of chemical pathology reports: the RCPAQAP Report Assessment Survey. Pathology 2016;48:357-62.

6. Royal College of Pathologists of Australasia (RCPA). Standards for Pathology Informatics in Australia (SPIA) (v3.0). Sydney: RCPA, 2017. Available at: https://www.rcpa.edu.au/Library/PractisingPathology/PTIS/APUTS-Downloads/Standards-and-Guidelines/ SPIA-(APUTS)-Standards-for-Pathology-Informatics-i. Accessed: 4 Apr 2018.

7. Australian Digital Health Agency. Establishment of Pathology Program. Available at: https://www.digitalhealth.gov.au/get-started- 
with-digital-health/what-is-digital-health/features-of-the-myhealth-record-system/pathology-program. Accessed: 1 Dec 2017.

8. Plebani M. Harmonization in laboratory medicine: requests, samples, measurements and reports. Crit Revs Clin Lab Sci 2016;53:184-96.

9. AS 4700.2 2012 Implementation of Health Level Seven (HL7) Version 2.4 Pathology and diagnostic imaging (diagnostics) Standards Australia 2012. Available at: http://www.e-health. standards.org.au/Home/Publications.aspx. Accessed: 4 Apr 2018.

10. Australian Digital Health Agency. eHealth Pathology Report v1.2 2016. Available at: https://www.digitalhealth.gov.au/implementation-resources/clinical-documents/EP-2242-2016. Accessed: 4 Apr 2018.

11. SAFER 2014. Test Results Reporting and Follow-Up. The Office of the National Coordinator for Health Information Technology.
Available at: https://www.healthit.gov/sites/default/files/ safer_test_results_reporting.pdf. Accessed: 1 Dec 2017.

12. O'Connor JD. Reducing post analytical error: perspectives on new formats for the blood sciences pathology report. Clin Biochem Revs 2015;36:7-20.

13. Zikmund-Fisher BJ, Scherer AM, Witteman HO, Solomon JB, Exe $\mathrm{NL}$, Tarini BA, et al. Graphics help patients distinguish between urgent and non-urgent deviations in laboratory test results. J Am Med Inform Assoc 2017;24:520-8.

14. Goetz, T. It's time to redesign medical data. TEDMED 2010. https://www.ted.com/talks/thomas_goetz_it_s_time_to_redesign_medical_data\#t-791508. Accessed: 18 Sep 2017.

15. Fraser CG. Biological variation from principles to practice. Washington, USA: AACC Press, 2001.

16. Kahneman D. Thinking fast and slow. New York: Farrar, Straus and Giroux, 2011. 